\title{
Statistical Analysis on Correlates of HIV Testing Outcomes Based on Ethiopia Demographic and Health Survey (EDHS) 2011 Data
}

\author{
Desalegn Dargasso Danna ${ }^{1, ~ *}$, Abebe Sorsa Badacho², Ayele Taye ${ }^{3}$ \\ ${ }^{1}$ Department of Statistics, College of Natural and Computational Sciences, Wolaita Sodo University, Sodo, Ethiopia \\ ${ }^{2}$ School of Public Health, College of Health Sciences and Medicine, Wolaita Sodo University, Sodo, Ethiopia \\ ${ }^{3}$ Department of Statistics, College of Natural Sciences, University of Gonder, Gonder, Ethiopia
}

Email address:

desdargaso@gmail.com (D. D. Danna), sorsabebe@gmail.com (A. S. Badacho)

${ }^{*}$ Corresponding author

\section{To cite this article:}

Desalegn Dargasso Danna, Abebe Sorsa Badacho, Ayele Taye. Statistical Analysis on Correlates of HIV Testing Outcomes Based on Ethiopia Demographic and Health Survey (EDHS) 2011 Data. Science Journal of Public Health. Vol. 6, No. 5, 2018, pp. 120-126. doi: $10.11648 /$ j.sjph.20180605.11

Received: February 16, 2018; Accepted: October 16, 2018; Published: October 27, 2018

\begin{abstract}
Voluntary Counseling and Testing (VCT) is the key entry point to prevention, care, and treatment and support services. The study aimed at describing the impacts of some pre-test counseling session factors on the status of HIV/AIDS. The data were taken from Ethiopia Demographic and Health Survey (EDHS 2011) conducted in 2011. For modeling purpose logistic regression was used and data were analyzed using SPSS version 20.00. Among 12,986 individuals encompassed in the study, 6,297 (48.5\%) were male. The prevalence of HIV/AIDS infection among Voluntary Counseling and Testing (VCT ) individuals was estimated to be $1.8 \%$ and varied by demographic, socio-economic and risk behavior characteristics of individuals. Determinant factors driving the prevalence of HIV among populations and wide variations in HIV prevalence exist across regions; highest in Gambela (7.5\%) and Addis Ababa (4.5\%). Sex, age at first sex, region, wealth index, educational level attained and occupation status were significant determinants of HIV testing. The odds of being HIV infected for female individual was about 36.9\% (95\% C.I. 1.043-1.796). The value of the odds for age at first sex indicates that the odds of age at first sex being HIV infected for aged greater than or equal to 35 years have had $32.7 \%$ more than those in the age group less than or equal to 18 years $(\exp (\widehat{\beta})=1.327$, and the odds of HIV infected for an individual in the age category $19-34$ were $83.1 \%$ less than an individual in the age group $\leq$ years $(\mathrm{OR}=0.831$, C.I. $=.534-1.294)$. Individuals found in Gambella region were 4.5 times HIV positive than Tigray region individuals $(\mathrm{OR}=4.460, \mathrm{P}=0.000$, C.I. $=2.457-8.098$ ). Clients whose wealth index is middle were about 4.041 times more likely HIV infected than poor clients $(\mathrm{OR}=4.041, \mathrm{P}=0.000$, C.I. $=2.556-6.389)$. Urban individuals are nearly 3.5 times likely HIV positive than rural individuals $(\mathrm{OR}=3.514, \mathrm{P}=0.000)$. High risk behaviour and low socio-economic position are found to be significantly related with HIV infection in Ethiopia.
\end{abstract}

Keywords: HIV Testing, Outcomes, VCT Services, Logistic Regression

\section{Introduction}

Worldwide, Human Immunodeficiency Virus/Acquired Immune- deficiency Syndrome (HIV/AIDS) poses an enormous challenge on the survival of mankind. The HIV pandemic remains the most serious of infectious disease challenges to public health. Promising developments have been seen in recent years in global efforts to address the
AIDS epidemic, including increased access to effective treatment and prevention programs. However, the number of people living with HIV and deaths due to AIDS continues to grow. Sub-Saharan Africa remains the most seriously affected region, with AIDS remaining the leading cause of death. More than two thirds (68\%) of all people HIV positive 
live in this region where more than three quarters $(76 \%)$ of all AIDS deaths in 2007 occurred [1].

The WHO data reveals that up until the end of 2016 more than 70 million people worldwide have been infected with HIV/AIDS of whom about 35 million have died of HIV/AIDS and related complications. Currently, SubSaharan African is home to about 36.7 million people living with the virus, making it the most affected in the world [2].

Ethiopia is one of the Sub-Saharan African countries hardhit by HIV/AIDS in all its manifestations HIV Counseling and Testing (HCT) is the key entry point to prevention, care, treatment and support services. Many people are reluctant to learn their HIV status when medical care for HIV related illnesses and psychosocial support does not exist, and in the absence of community support and legal protection when they face discrimination and social marginalization [3]. HIV counseling and testing is one of the key strategies in the HIV/AIDS prevention and control programs in Ethiopia. However utilization of this service is very low [4].

Ethiopian population growth has slowed dramatically or stopped due to HIV and AIDS. A logistic regression and correlation between HIV positive towards AIDS, high risk factors leading to exposure to HIV infection was adopted with selected variables. The study confirmed that the high level of HIV positive and the poor health was highly affected with socioeconomic and demographic factors [5].

There are three types of HIV testing in the country: Clientinitiated, or voluntary counseling and testing Providerinitiated testing and counseling

Mandatory HIV screening

In Ethiopia, One of serious problem acknowledged and all the concerned bodies making efforts to control the spread of the epidemic through different mechanisms. One of many different strategies designed for prevention and control of the disease is providing voluntary HIV counseling and testing (VCT) [6]. VCT is an intentionally recognized, effective and important strategy for both preventive and care studies. It is cost effective strategy for facilitating behavioral change and is also important entry point for care and support for those whose test result become positive. It also provides people with an opportunity to learn and accept their HIV testing outcomes in a confidential environment with counseling and referral for ongoing emotional support and medical care. People whose test outcome is positive can benefit from earlier by appropriate medical including ART treatment and HIV associated illness, social support, emotional and spiritual care. The high prevalence of HIV/AIDS in Ethiopia requires an extensive demand for VCT services. This demand is given due to attention from government and nongovernment organizations.

The 2016 Ethiopian Demographic Health Survey (DHS) reveals that around $56 \%$ of the women and $55 \%$ of the men among the surveyed household have never been tested for $\mathrm{HIV}$, an indication the current number of HIV positives in the country could be a lot more had all the population been tested. And, despite the existence of the large number of people living with HIV/AIDS, only $72 \%$ of them are thought to be aware that they are living with the virus; the remaining $28 \%$ think they are not infected [7].

The HIV/AIDS situation in Ethiopia continues to be characterized by a low intensity, mixed epidemic with significant heterogeneity across geographic areas and defined by independent self -sustaining HIV transmission. Per spectrum preliminary estimate by PEPFAR, January 2017, adult HIV prevalence in Ethiopia in 2016 was estimated to be $1.1 \%$. There is substantial prevalence variation by region (6.6\% in Gambella, 5.0\% in Addis Ababa, and 0.7\% in Southern Nations, Nationalities and Peoples' (SNNPR) region). The HIV epidemic in Ethiopia is primarily associated with areas of urban concentration $(5.1 \%$ in cities above 50 thousand compared to $3.1 \%$ in smaller cities and $0.6 \%$ in rural areas) and proximity to major transport corridors. Those living within five kilometers of a major road have HIV prevalence rates that are four - times higher than those who live further away [8].

Today, Ethiopia has made progress in reducing the number of HIV/AIDS nationally, but the observed changes are not sufficient enough compared to the desired goals of the response against the epidemic. Given the size of population and the magnitude of the damage inflicted, it will take us a number of years to see significant declines in HIV prevalence and incidence with concerted and sustained efforts and also there are advances in the availability, accessibility and utilization of HIV/AIDS prevention, care, support and treatment services and improvements in the managements of the epidemic and the increasing resource availability, still need more efforts to control the problem. Investigating the existence of significant associations between the above factors and HIV infection can provide evidence for informed prevention mechanisms. Furthermore, modeling the probability of contracting HIV is helpful to identify a group with higher chance of getting the virus and take evidence based on prevention measure, HCT (HIV Counseling and testing) and identifying risk factors for the infection of HIV. Therefore this study focused to examine a range of potential factors, which have significant effect on HIV testing outcomes of an individual in both rural and urban areas in Ethiopia based on EDHS data 2011 [9].

\section{Methodology}

\subsection{Source of Data}

This research was utilized the 2011 Ethiopian Demographic and Health Survey (EDHS) data conducted by Central Statistical Agency (CSA) in 2011. The survey is the third comprehensive and nationally representative population and health survey. The 2011 Ethiopian Demographic and Health Survey is designed to provide estimates for the health and demographic variables of interest for the following domains; Ethiopia as a whole; urban and rural areas of Ethiopia (each as a separate domain) and 11geographic areas (9 regions and 2 city 
administrations) and it was a nationally representative survey of women aged 15-49 from 17,817 households from 624 clusters (187 urban and 437 rural) areas throughout Ethiopia. Multistage cluster sample based on the 1994 Population and Housing Census sample frame and was designed to obtain information on the basic indicators of the health and demographic variables. it includes all geographic areas (nine regions namely: Tigray, Affar, Amhara, Oromiya, Somali, Benishangul-Gumuz, Southern Nations, Nationalities and Peoples (SNNP), Gambela and Harari regional states and two city administrations ; (Addis Ababa and Dire Dawa). In this data sample was selected using a stratified, two-stage cluster design and EAs were the sampling units for the first stage. The sample included 624 Enumeration areas (EAs), 187 in urban areas and 437 in rural areas.

\subsection{Measurement}

Outcome variable dependent / response variable is the dichotomous variable "HIV testing outcome of the respondent" (HIV positive $=1$, HIV negative $=0$ ).

\subsection{Model Selection}

As described by Hosmer and Lemeshow there are certain steps that can be followed in the selection of variables for a logistic regression model. First, the selection process should begin with a careful univariate analysis of each the variables. For this purpose, the Pearson's chi-square and/or the likelihood ratio chi-square tests was used. Up on completion of the univariate analysis variables were selected for the multiple logistic regression analysis. Variables whose univariate test has a p-value less than 0.05 considered as a candidate for multiple logistic regression analysis. Finally, following the fit of the multiple logistic regression models, the importance of each variable included in the model verified using examination of the Wald statistic for each of the variables and the comparison of each estimated coefficient with the coefficient from the univariate model containing only that variable [10].

\section{Results}

\subsection{Summary Statistics}

Total of 12,986 individuals received VCT services within the surrounding ten $\mathrm{km}$ radius from the cluster for each region were included in this study. Among those individuals $51.5 \%$ were females. In relation to age at first sex, $49.6 \%$ of respondent were greater than or equal to 35 years old. The majority of individuals $(80.7 \%)$ were from rural. $58.0 \%$ had no formal education. The majority of respondents $(63.9 \%)$ were unemployed. According to regional variation of Ethiopia, large number of individuals covered in this study were from Affar, SNNP, Amhara, Oromiya, Addis Ababa, Somali and Tigray $(14.8 \%, 14.5 \%, 13.6 \%, 13.1 \%, 8.3 \%$, $7.7 \%$ and7.1) respectively. In relation to Religion, the majority of individuals, about $42.1 \%$, were from Orthodox, $37.8 \%$ from Muslim and 20.1\% were protestant.

Prevalence of HIV infection varies by the Socio-economic and Demographic Characteristic of the VCT individuals. Sero-Positivity is high among females than males. According to the findings, $1.5 \%$ of the male and $2.0 \%$ of the female clients were found to be HIV positive. HIV prevalence appeared to be significantly lower (1.4\%) among individuals 19-34 years than in individuals at older age group of first sexual intercourse. Individuals aged greater than or equal to 35 years exhibited higher prevalence of HIV infection (2.1\%) and those whose age at first sexual inter course less or equal to 18 years $(1.5 \%)$. Respondents in the highest wealth index have the highest HIV prevalence; that means wealth index increases from poor to rich, individuals being HIV positive also increases. HIV prevalence is higher in urban (4.8 percent) areas than in rural areas (1.0 percent). A lower proportion of respondents who attended less educational level (No formal education) than respondents who attended Primary and above education level. A significantly higher prevalence was found among individuals who were employed $(2.1 \%)$ unemployed (1.6\%). Among regions, HIV prevalence is highest in Gambela (7.5\%), Addis Ababa (4.5\%) and Dire Dawa (3.0\%). Prevalence of HIV is relatively highest among Muslim (1.8 percent) and Orthodox (1.7 percent). Regarding to age, individuals in 26-35 age group was highly affected by the Virus (Table 1).

Table 1. Cross tabulation of Response variable with Predictor variables.

\begin{tabular}{|c|c|c|c|c|c|c|c|}
\hline \multirow{3}{*}{ Variables } & \multirow{3}{*}{ Category } & \multirow{3}{*}{ Frequency $(\%)$} & \multicolumn{4}{|c|}{ HIV testing outcomes of respondents } & \multirow{3}{*}{$\begin{array}{l}\mathrm{X}^{2} \\
(\mathrm{P} \text { Value })\end{array}$} \\
\hline & & & \multicolumn{2}{|c|}{ HIV negative } & \multicolumn{2}{|c|}{ HIV positive } & \\
\hline & & & Count & Row N \% & Count & Row N \% & \\
\hline \multirow{2}{*}{ Sex } & Male & $6297(48.5)$ & 6198 & 98.5 & 94 & 1.5 & \multirow{2}{*}{.027} \\
\hline & Female & 6689 (51.5) & 6548 & 98.0 & 134 & 2.0 & \\
\hline \multirow{5}{*}{ Age } & $<5$ years & $2182(16.8)$ & 2141 & 98.3 & 38 & 1.7 & \multirow{5}{*}{936} \\
\hline & $5-15$ & $4151(32.0)$ & 4078 & 98.4 & 67 & 1.6 & \\
\hline & $16-25$ & 2398 (18.5) & 2355 & 98.3 & 41 & 1.7 & \\
\hline & $26-35$ & 1615 (12.4) & 1583 & 98.0 & 32 & 2.0 & \\
\hline & $\geq 36$ years & $2639(20.3)$ & 2588 & 98.1 & 50 & 1.9 & \\
\hline \multirow{3}{*}{ Age at firs sex } & $\leq 18$ years & $4346(33.5)$ & 4275 & 98.5 & 66 & 1.5 & \multirow{3}{*}{.040} \\
\hline & $19-34$ & $2178(16.8)$ & 2148 & 98.6 & 30 & 1.4 & \\
\hline & $\geq 35$ years & 6443 (49.6) & 6304 & 97.9 & 132 & 2.1 & \\
\hline
\end{tabular}




\begin{tabular}{|c|c|c|c|c|c|c|c|}
\hline \multirow{3}{*}{ Variables } & \multirow{3}{*}{ Category } & \multirow{3}{*}{ Frequency $(\%)$} & \multicolumn{4}{|c|}{ HIV testing outcomes of respondents } & \multirow{3}{*}{$\begin{array}{l}\mathrm{X}^{2} \\
\text { (P Value) }\end{array}$} \\
\hline & & & \multicolumn{2}{|c|}{ HIV negative } & \multicolumn{2}{|c|}{ HIV positive } & \\
\hline & & & Count & Row N \% & Count & Row N \% & \\
\hline \multirow{3}{*}{ Wealth index } & Poor & $5886(45.3)$ & 5839 & 99.3 & 43 & 0.7 & \multirow{3}{*}{.000} \\
\hline & Middle & $2079(16.0)$ & 2030 & 97.9 & 43 & 2.1 & \\
\hline & Rich & $5021(38.7)$ & 4877 & 97.2 & 142 & 2.8 & \\
\hline \multirow{2}{*}{ Residence } & Urban & $2504(19.3)$ & 2384 & 95.2 & 120 & 4.8 & \multirow{2}{*}{.000} \\
\hline & Rural & $10482(80.7)$ & 10362 & 99.0 & 108 & 1.0 & \\
\hline \multirow{2}{*}{ Educational level } & No formal education, & $7508(57.8)$ & 7398 & 98.7 & 100 & 1.3 & \multirow{2}{*}{.000} \\
\hline & Primary and above & $5458(42.0)$ & 5328 & 97.7 & 128 & 2.3 & \\
\hline \multirow{2}{*}{ occupation } & Unemployed & $8302(63.9)$ & 8165 & 98.4 & 130 & 1.6 & \multirow{2}{*}{.027} \\
\hline & Employed & $4670(36.0)$ & 4567 & 97.9 & 98 & 2.1 & \\
\hline \multirow{4}{*}{ Region } & Tigray & $924(7.1)$ & 907 & 98.2 & 17 & 1.8 & \multirow{4}{*}{.000} \\
\hline & Affar & $1924(14.8)$ & 1901 & 99.0 & 20 & 1.0 & \\
\hline & Amhara & $1771(13.6)$ & 1750 & 98.9 & 20 & 1.1 & \\
\hline & Oromiya & $1698(13.1)$ & 1683 & 99.4 & 11 & 0.6 & \\
\hline \multirow{10}{*}{ Religion } & Somali & $995(7.7)$ & 982 & 98.8 & 12 & 1.2 & \multirow{10}{*}{.866} \\
\hline & Benishangul-Gumuz & $835(6.4)$ & 821 & 98.4 & 13 & 1.6 & \\
\hline & SNNP & $1882(14.5)$ & 1872 & 99.5 & 10 & 0.5 & \\
\hline & Gambela & $544(4.2)$ & 503 & 92.5 & 41 & 7.5 & \\
\hline & Harari & $792(6.1)$ & 772 & 97.6 & 19 & 2.4 & \\
\hline & Addis Ababa & $1073(8.3)$ & 1037 & 95.5 & 49 & 4.5 & \\
\hline & Dire Dawa & $548(4.2)$ & 518 & 97.0 & 16 & 3.0 & \\
\hline & Muslim & $4911(37.8)$ & 4812 & 98.2 & 90 & 1.8 & \\
\hline & Orthodox & $5463(42.1)$ & 5369 & 98.3 & 93 & 1.7 & \\
\hline & Other & $2612(20.1)$ & 2565 & 98.3 & 45 & 1.7 & \\
\hline
\end{tabular}

\subsection{Logistic Regression Analysis}

The forward stepwise Likelihood Ratio method is used to select the best variables. Fitting a model to a set of data first entails estimating the unknown parameters in the model. In order to fit a linear logistic model to a given set of data, the $\mathrm{k}+1$ unknown parameters $\beta_{0}, \beta_{1}, \beta_{2}, \ldots, \beta_{\mathrm{k}}$ have been estimated. These parameters are readily estimated using the method of maximum likelihood. This procedure revealed that the variables sex, age at first sex, wealth index, occupation status, residence and region were important predictors of HIV testing outcomes of an individual. Goodness and fit of model were carried out by assessing and diagnose the goodness of fit of the model. Likelihood Ratio Test was carried out. The likelihood ratio test statistic used to determine the overall significance of logistic model.

The initial model is called initial chi-square which is -2LL for the model, which accepts the null hypothesis that all the $\beta$ coefficients are zero $\left(\mathrm{H}_{0}: \beta=0\right)$. This implies that none of the independent variable is linearly related to the log odds of the dependent. The model chi-square thus tests the null hypothesis that all population logistic regression coefficients except the constant are zero. The degrees of freedom in this test equal the number of terms in the model minus 1 (for the constant). This is the same as the difference in the number of terms between the two models, since the null model has only one term. Model chi-square measures the improvement in the fit that the explanatory variables make compared to the null model. Model chi-square is a likelihood ratio test, which reflects the difference between the error of not knowing the independent variables (initial chi-square) and error when the independent variables are included in the model (deviance).

Likelihood ratio for full model is 2020.254and 2292.945 for restricted model. Model chi-square is obtained by the difference between Initial and full model (that is, $2292.945-2020.254=272.691$ ) with P-value (sig.) $=0.000$ which indicates significant decrease in deviance thereby implying a good fit of the model.

The model predicting HIV testing outcome of HIV positive individuals correctly classifies 75 out of 228 , or $\left(\frac{75}{228}\right) * 100 \%=32.9 \%$ of $\mathrm{HIV}$ positive individual. The "Overall Percentage" of $92.0 \%$. Using the classification table and the model chi-square goodness fit test model was statistically significant.

\subsection{Checking for Influential Observations}

Adequacy of the fitted model was checked for possible presence and treatment of outliers and influential values. The diagnostic test results for detection of outliers and influential values were given (Table 2).

Analog of Cook's influence statistics, Leverage value and DFBETA for model parameters including the constant term were both less than unity. Small values of Analog of Cook's influence statistics, Leverage value and DFBETA $A_{s}$ indicate that the final model fits the data. 
Table 2. Results of diagnostic tests for outliers and influential values.

\begin{tabular}{|c|c|c|}
\hline & Minimum & Maximum \\
\hline Analog of Cook's influence statistics & .00000 & .19045 \\
\hline Leverage value & .00022 & .02396 \\
\hline DFBETA for constant & -.05162 & .10024 \\
\hline DFBETA for Age at first $\operatorname{sex}(2)$ & -.01754 & .03664 \\
\hline DFBETA for Age at first sex(3) & -.01623 & .00911 \\
\hline DFBETA for Sex(1) & -.01301 & .00883 \\
\hline DFBETA for Region(2) & -.07070 & .05865 \\
\hline DFBETA for Region(3) & -.06269 & .05691 \\
\hline DFBETA for Region(4) & -.06357 & .10021 \\
\hline DFBETA for Region(5) & -.06613 & .09033 \\
\hline DFBETA for Region(6) & -.06405 & .08885 \\
\hline DFBETA for Region(7) & -.06781 & .10819 \\
\hline DFBETA for Region(8) & -.06813 & .03487 \\
\hline DFBETA for Region(9) & -.06905 & .06298 \\
\hline DFBETA for Region(10) & -.07273 & .04408 \\
\hline DFBETA for Region(11) & -.06494 & .07416 \\
\hline DFBETA for Wealth index(1) & -.03742 & .05718 \\
\hline DFBETA for Wealth index(2) & -.03042 & .04761 \\
\hline DFBETA for Residence(1) & -.04742 & .05504 \\
\hline DFBETA for Occupation status(2) & -.01150 & .01265 \\
\hline
\end{tabular}

\subsection{Interpretation of the Results of Binary Logistic Regression}

The values of the Wald statistic for individual $\beta$ coefficients support that the estimated values $\left(\hat{\beta}_{\mathrm{i}}^{\prime} \mathrm{s}\right)$ are significantly different from zero at $\alpha=0.05$ level of significance for all selected factors. The estimated coefficients $\left(\hat{\beta}_{\mathrm{i}}^{\prime} \mathrm{s}\right)$ for the factors in the final model, their standard error the odds ratio corresponding each estimated coefficient $\left(\widehat{\beta}_{\mathrm{i}}\right)$ were obtained by the procedures of Forward Stepwise (Likelihood Ratio). The final logistic regression model includes only those significant variables.

The stepwise selection proceeded by entering the variable; Variable(s) entered on step 1: Residence, Variable entered on step 2: Region, Variable entered on step 3: Wealth index, Variable entered on step 4: occupation, Variable entered on step 5: Sex, Variable entered on step 6: Age at first sex. The likelihood ratio test criterion is used to select and remove variables at each step of the procedures. The $5 \%$ level of significance is used for entry of variables. The reference category is the first category of sex, age at first sex, wealth index region and occupation status but it is the last category of Residence. The significance of the Wald statistic indicates the importance of the predictor variables in the model.

The logistic regression model indicates that HIV testing outcome of an individual is affected by many factors. sex, age at first sex, region, wealth index, occupation and residence were found to have significant effect on HIV infection at significance level of 5\%. Prevalence of HIV infection varies by sex. The odds of being HIV infected for female individual is about $36.9 \%$ more than male clients and its effect is statistically significant. The value of the odd ratio $\exp (\widehat{\beta})=1.327$, for age at first sex indicates that the odds of being HIV infected for an individual aged greater than or equal to 35 years have $32.7 \%$ more than those in the age group less than or equal to 18 years and the odds of HIV infected for an individual in the age category $19-34$ are $83.1 \%$ less than an individual in the age group $\leq$ 18 years $(\mathrm{OR}=0.831, \mathrm{C} . \mathrm{I}=.534-1.294) \quad$. Regional variation, the reference category is Tigray. The odds of HIV infection has decreased by a factor of 0.526 for individuals living in Afar compared to those in Tigray controlling for other variables in the model. Similarly, the odds of HIV infection has decreased by a factor of 0.517 for individuals living in Amhara compared to those in Tigray. The odds of $\mathrm{HIV}$ infection in Gambela region is found to be 4.46 (95\% C.I 2.45 - 8.098) times that of Tigray region., implying that of individuals in SNNP region displayed a lower chance to be HIV positive as compared to Tigray region individuals. Clients whose wealth index is middle were about 4.041 times more likely HIV infected than poor clients. The odds of being HIV infected employed individual was about $45.0 \%$ more than those individuals who were unemployed. The positive model odds ratio (3.514) for urban individuals indicates that the prevalence of HIV infection was higher than rural ( Table 3) 
Table 3. Binary Logistic Regression of Statistical analysis of correlates of HIV testing outcomes based on Ethiopia Demographic and Health Survey (EDHS) 2011 data.

\begin{tabular}{|c|c|c|c|c|c|c|c|c|c|}
\hline \multirow{2}{*}{ Variables } & \multirow{2}{*}{ Category } & \multirow{2}{*}{ B } & \multirow{2}{*}{ S.E. } & \multirow{2}{*}{ Wald } & \multirow{2}{*}{ Df } & \multirow{2}{*}{ Sig. } & \multirow{2}{*}{$\operatorname{Exp}(B)$} & \multicolumn{2}{|c|}{$95 \%$ C.I for $\operatorname{EXP}(B)$} \\
\hline & & & & & & & & Lower & Upper \\
\hline \multirow{3}{*}{ Sex } & $1=$ Male (Ref) & & & & & & & & \\
\hline & $2=$ Female & .314 & .139 & 5.122 & 1 & .024 & 1.369 & 1.043 & 1.796 \\
\hline & & & & 6.793 & 2 & .033 & & & \\
\hline \multirow{3}{*}{ Age at first sex } & $1=\leq 18($ Ref $)$ & & & & & & & & \\
\hline & $2=19-34$ & -.185 & .226 & .670 & 1 & .413 & .831 & .534 & 1.294 \\
\hline & $3=\geq 35$ & .283 & .155 & 3.350 & 1 & .067 & 1.327 & .980 & 1.798 \\
\hline \multirow{13}{*}{ Region } & & & & 123.029 & 10 & .000 & & & \\
\hline & $1=$ Tigray $($ Ref $)$ & & & & & & & & \\
\hline & $2=$ Afar & -.643 & .341 & 3.548 & 1 & .060 & .526 & .269 & 1.026 \\
\hline & $3=$ Amhara & -.660 & .336 & 3.860 & 1 & .049 & .517 & .268 & .998 \\
\hline & 4=Oromiya & -1.196 & .393 & 9.262 & 1 & .002 & .302 & .140 & .653 \\
\hline & $5=$ Somali & -.525 & .384 & 1.871 & 1 & .171 & .591 & .279 & 1.255 \\
\hline & $6=$ Ben-Gumuz & -.005 & .378 & .000 & 1 & .990 & .995 & .475 & 2.086 \\
\hline & $7=\mathrm{SNNP}$ & -1.243 & .407 & 9.341 & 1 & .002 & .288 & .130 & .640 \\
\hline & $8=$ Gambela & 1.495 & .304 & 24.145 & 1 & .000 & 4.460 & 2.457 & 8.098 \\
\hline & 9=Hareri & -.037 & .345 & .012 & 1 & .914 & .964 & .490 & 1.896 \\
\hline & $10=$ Addis Ababa & -.263 & .312 & .708 & 1 & .400 & .769 & .417 & 1.418 \\
\hline & 11=Dire-Dawa & -.181 & .361 & .252 & 1 & .616 & .834 & .411 & 1.693 \\
\hline & & & & 35.706 & 2 & .000 & & & \\
\hline \multirow{3}{*}{ Wealth index } & $1=$ Poor(Ref) & & & & & & & & \\
\hline & 2=Middle & 1.396 & .234 & 35.706 & 1 & .000 & 4.041 & 2.556 & 6.389 \\
\hline & $3=$ Rich & .764 & .235 & 10.565 & 1 & .001 & 2.147 & 1.354 & 3.404 \\
\hline \multirow{2}{*}{ Residence } & $1=$ Urban & 1.257 & .216 & 33.934 & 1 & .000 & 3.514 & 2.302 & 5.363 \\
\hline & $2=\operatorname{Rural}(\operatorname{Ref})$ & & & & & & & & \\
\hline \multirow{2}{*}{ Occupation } & $0=$ No (Ref) & & & & & & & & \\
\hline & $1=$ Yes & .371 & .138 & 7.199 & 1 & .007 & 1.449 & 1.105 & 1.901 \\
\hline Constant & -4.463 & .333 & 179.681 & 1 & .000 & .012 & & & \\
\hline
\end{tabular}

**Ref indicates the reference category

a. Variable(s) entered on step 1: Residence.

b. Variable(s) entered on step 2: Region

c. Variable(s) entered on step 3: Wealth index.

d. Variable(s) entered on step 4: occupation

e. Variable(s) entered on step 5: Sex.

f. Variable(s) entered on step 6: Ag at first sex.

\section{Discussion}

This study was undertaken to determine the effect of some demographic, socio-economic and HIV related risk behavior variables on the HIV testing Outcomes in Ethiopia. Based data from the Ethiopia Demographic and Health Survey (EDHS) 2011 [9].

Based on the result of this study, about $1.5 \%$ of the male individual and $2.0 \%$ of the female individuals were found to be HIV positive. This pattern of female exposure to HIV infection may be due to the biological factors as well as low socio-economic status of women and other cultural influences. A similar study conducted in Urban indicated that $7.1 \%$ of the male and $9.7 \%$ of the female individuals were HIV positive [10]. The finding of this study shows that residential differences had a significant impact on the prevalence of HIV/AIDS. Respondents who lived in urban areas were more likely to be HIV positive than rural respondents. With regard to wealth index rich individuals highly affected by the virus $(2.8 \%)$ followed by individuals who were categorized in middle class $(2.1 \%)$ and poor individuals $(0.7 \%)$. The HIV prevalence was estimated at
$6.2 \%$ (9.58\% for urban areas and $5.04 \%$ for rural) in 2009 [11]. Occupation status of an individual is another important explanatory variable affecting HIV testing outcome of an individual. The finding of this study shows that individuals who are not occupation (1.6 percent) are less likely to be HIV positive than individuals who were employed ( 2.1 percent). But, the study by in urban Hawassa showed that, HIV prevalence appeared to be highest among individuals who were unemployed than those who were employed [12]. With regard to regional variations, HIV prevalence is highest in Gambella (7.5 percent) and Addis Ababa (4.5) followed by Dire Dawa and Harari (3.0 and 2.4 percent) respectively. According to research conducted in Ethiopia, Gambella region was characterized by a relatively higher HIV prevalence than any other region.

\section{Conclusion}

The main aim of Voluntary Counseling and Testing (VCT) is to prevent and control HIV occurrence, it would be essential to study the factors that can improve the performance of VCT.

The demographic and socio-economic profile of Voluntary 
Counseling and Testing (VCT) individuals in Ethiopia showed that the age 26-35 years to be the most affected age group by HIV/AIDS. Pointing this age group would greatly reduce the risk of HIV/AIDS. Female clients were more likely to be HIV positive than males. This pattern of female vulnerability to HIV/AIDS infection in Ethiopia is commonly seen in many other studies. Perhaps due to the biological factors as well as the prevailing low socio economic status of women and other cultural influences, targeting women for HIV prevention and control is required. There was association between HIV infection and one's occupation status. That is, employed individuals are among the highly affected parts of the society. Highest Educational levels of individuals have a significant influence on HIV infection, as the level of education increases the prevalence of HIV increases.

\section{Recommendation}

The high prevalence of HIV/AIDS in Ethiopia calls for an extensive demand for Voluntary Counseling and Testing (VCT) services. This demand is given outstanding consideration from government and non-government organizations.

\section{Limitation of the Study}

In this study only positive HIV status and negative HIV status are included. Individuals whose results are indeterminate are excluded from the study.

Data problem - Some important variables are not included because of missing values and non-responses.

The data, used in this study are from the EDHS 2011. Thus, the results may not necessarily reflect the current situation of Ethiopia.

\section{References}

[1] UNAIDS/WHO (2007). Global Summary of the AIDS Epidemic Update.
[2] Addis standard: Analysis: HIV/AIDS is surging in Ethiopia, again / October 6, 2017.

[3] Ministry of Health (2007). Federal HIV/AIDS in Ethiopia. Disease Prevention and Control Department, 6thEdition: Addis Ababa, Ethiopia.

[4] Godif M, Assefa H, Alemayehu M, Terefe M (2015) Factors Associated with HIV Counseling and Testing among Males and Females in Ethiopia: Evidence from Ethiopian Demographic and Health Survey Data. J AIDS Clin Res 6: 429. doi:10.4172/2155-6113.1000429.

[5] A Sathiya Susuman. HIV/AIDS in Ethiopia: Health View; Journal of Asian and African studies 1- 12 March 2015; University of the Western Cape DOI: $10.1177 / 0021909615570957$.

[6] FMOH (2007). Federal HIV/AIDS Prevention and Control Office. Guidelines for HIV councelling and Testing in Ethiopia.

[7] The 2016 Ethiopia Demographic and Health Survey (2016 EDHS) was implemented by the Central Statistical. Agency (CSA) from January 18, 2016, to June 27.

[8] Ethiopia; Country/Regional Operational Plan (COP/ROP) 2017. Strategic Direction Summary. April 21, 2017.

[9] Central Statistical Agency (Ethiopia), Macro ORC (2012) Ethiopia Demographic and Health Survey 2011. Addis Ababa, Ethiopia.

[10] Demeke Ertiro (2010). Analysis of HIV Status in Relation to Some Demographic and HIV Related Risk Behavior Factors: The Case of OSSA Free Standing VCT Center in Urban Hawassa. MSc thesis, Statistics Department, Hawassa University, Ethiopia.

[11] Melaku Dejene (2012). Modelling HIV/AIDS Epidemics in Ethiopia by Incorporating the Effects of ART and Population Change Over Time. MSc thesis, Statistics Department, Hawassa University, Ethiopia.

[12] Hosmer, D. W. and Leme show, S. (1989): Applied Logistic Regression, NewYork: John Wiley. 